Research Paper

\title{
Neoadjuvant chemotherapy followed by minimally invasive esophagectomy versus primary surgery for management of esophageal carcinoma: a retrospective study
}

\author{
Yan Zheng ${ }^{*}$, Yin $\mathrm{Li}^{*}, \bowtie$, Xianben Liu, Ruixiang Zhang, Zongfei Wang, Haibo Sun, Shilei Liu \\ Department of Thoracic Surgery, The Affiliated Cancer Hospital of Zhengzhou University, Henan Cancer Hospital, Zhengzhou, Henan 450008, P. R. China \\ "Yan Zheng, and "Yin Li contributed equally to this article. \\ $\square$ Corresponding author: Li Yin, MD, PhD, Department of Thoracic Surgery, The Affiliated Cancer Hospital of Zhengzhou University, Henan Cancer Hospital, \\ Zhengzhou, Henan 450008, P. R. China. Tel.: +86 371 65587226; E-mail address: liyin0825@hotmail.com \\ (c) Ivyspring International Publisher. This is an open access article distributed under the terms of the Creative Commons Attribution (CC BY-NC) license \\ (https://creativecommons.org/licenses/by-nc/4.0/). See http://ivyspring.com/terms for full terms and conditions.
}

Received: 2018.08.19; Accepted: 2019.01.04; Published: 2019.01.29

\begin{abstract}
There is no consensus about the combined therapeutic strategy for esophageal squamous cell carcinoma in China. The quality control and standardization of surgery procedures were far from satisfactory in past neoadjuvant chemotherapy trials, which may underestimate the survival benefits. Therefore, we tried to evaluate the survival benefit of paclitaxel plus platinum followed by minimally invasive esophagectomy with total two-field lymphadenectomy patterns versus primary surgery. Between 06/2011 and 12/2014, there were 279 consecutive patients who underwent minimally invasive esophagectomy with total two-field lymphadenectomy; 83 received neoadjuvant chemotherapy and 196 primary surgery. Propensity score matching was used to compare neoadjuvant chemotherapy patients and 76 matched primary surgery patients. Effectiveness of neoadjuvant chemotherapy, adverse events, complications after the operation, and survival rates were evaluated. After propensity score matching, and compared with primary surgery, neoadjuvant chemotherapy was significantly associated with a better survival $(P=0.049)$. The overall clinical response rate of neoadjuvant chemotherapy was $\mathbf{7 7 . 1} \%$. The pathological response rate was $20.5 \%$. There was no significant difference in complication rates between two groups. Neoadjuvant chemotherapy with paclitaxel plus platinum followed by minimally invasive esophagectomy and total two-field lymphadenectomy have better OS over the primary surgery without serious adverse events.
\end{abstract}

Key words: minimally invasive esophagectomy, neoadjuvant chemotherapy, survival

\section{Background}

More than $50 \%$ of worldwide esophageal squamous cell carcinoma (ESCC) patients are located in China [1]. There is no consensus about the combined therapeutic strategy for ESCC in China due to lack of randomized controlled trials (RCT). Although the multidisciplinary therapeutic strategies have been explored for many years in both western countries [2-7] and Japan [8], the treatment is quite different from region to region based on the results of local RCTs.

The North American intergroup trial for ESCC (Radiation Therapy Oncology Group, RTOG Trial
8911 or USA Intergroup 113) [2, 3] suggested that there was no survival benefit from NAC for ESCC, whereas the Japan Clinical Oncology Group trial (JCOG9907) [8] showed promising results for the same procedure. The survival benefit of neoadjuvant chemotherapy (NAC) for ESCC remains controversial worldwide [9, 10]. Due to contradictory evidence outside China and the lack of RCT in China, we collected data from published articles and found the quality control of surgical procedures in the RCTs was poor [11]. At that time, lymph node sampling and transthoracic esophagectomy, transhiatal esophagec- 
tomy were acceptable in some trials. Surgery was the only local control method that existed in the NAC strategy. Different surgical procedures and ranges of lymphadenectomies may mask the benefits of chemotherapy and significantly affect the survival rate.

In light of past RCTs [2-5, 8], we have designed a retrospective analysis in our institute. The main concern of this study was to declare the survival benefit of NAC-platinum plus paclitaxel (TP) over the primary surgery based on quality controlled and standardized surgical procedure. Minimally invasive esophagectomy with total two-field lymphadenectomy was the only used surgical procedure.

\section{Methods}

\section{Inclusion criteria}

The Ethics Review Committee of Henan Cancer Hospital/The affiliated Cancer Hospital of ZhengZhou University approved this study officially. The approval number is 2016ct081. Patients' personal data were obtained. The consecutive patients were retrospectively evaluated between 06/2011 and 12/2014. The inclusion criteria were as follows: i) Thoracic ESCC; ii) Clinical staging T2-4aN0-2; iii) MIE with total two-field lymphadenectomy; iv) With follow up, without clinical missing data. The TNM Classification of Malignant Tumors 7th edition was adopted for the clinicopathological profiles [12].

\section{Neoadjuvant Chemotherapy}

NAC comprised 2 cycles. Cisplatin or nedaplatin plus paclitaxel were repeated once every 3 weeks. A dose of $175 \mathrm{mg} / \mathrm{m} 2$ paclitaxel was given by intravenous drip infusion on days 1 and 8. Cisplatin or nedaplatin was administered at a dose of 75 $\mathrm{mg} / \mathrm{m} 2$ by continuous infusion on days $2-4$.

\section{Surgical procedure}

All patients received a right thoracolaparoscopic esophagectomy and at least 2-field lymph node dissections. Cervical lymphadenectomy was performed when suspicious lymph node metastasis was reported on a color ultrasound. First, the patient was placed in a left semi-prone-position. The thoracic esophagus was mobilized and the lymph nodes were dissected. The regional lymph nodes included the mediastinal lymph nodes (left recurrent laryngeal never, right recurrent laryngeal nerve, paraesophageal, paratracheal, subcarinal, supradiaphragmatic and posterior mediastinal lymph nodes). Then the position was modified to a supine position. A $2-3 \mathrm{~cm}$ left cervical incision was made. Muscle sparing methods and blunt dissection were used to expose the cervical esophagus. In the abdominal part, laparoscope was used for gastric mobilization. A 3-cm gastric tube was made with linear staplers (TLC, Ethicon, USA) and perigastric lymph nodes (celiac, left gastric artery, common hepatic artery and splenic artery lymph nodes were dissected. Finally, the gastric conduit was delivered to the neck to be sewn with the distal esophagus by using a hand-sewn 3 layered anastomosis (Li's anastomosis) [13]. Figure 1 showed the standard extent of lymph nodes dissection.
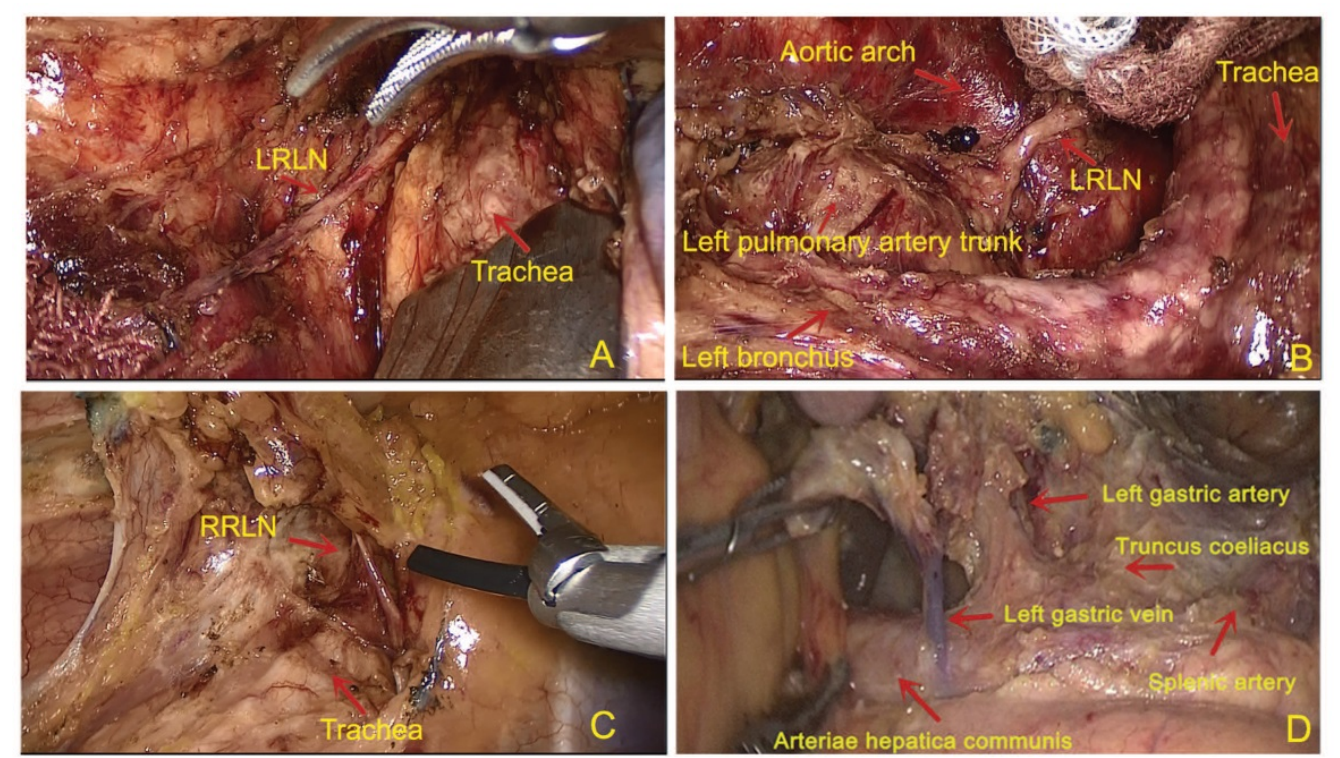

Figure 1. The graph of standard lymph node dissection. (A) and (B) exposure of the left recurrent laryngeal nerve (RLN). The lymph nodes from thyroid gland and the $L N$ s along the left RLN were removed using endoscopic scissors; (C) The region of lymph nodes dissection along the right recurrent laryngeal nerve (RLN); (D) The region of lymph nodes dissection in abdominal cavity. 


\section{Clinical and Pathological Tumor Effects}

Preoperational investigations included esophagography, esophageal endoscopic ultrasound, esophagoscopy, contrast-enhanced thoracic CT scan, abdominal ultrasound, brain MRI, cervical color ultrasound, and Emission Computed Tomography (ECT). If a patient accepted, positron emission tomography-CT (PET/CT) would be adopted instead of chest CT, abdominal ultrasound, cervical color ultrasound and ECT. No patients have repeated PET scan and endoscopy after induction. The Response Evaluation Criteria in the Solid Tumors [14] were used to evaluate the clinical tumor responses. Radiographic criteria for positive lymph nodes was that the shortest diameter of lymph nodes $>1 \mathrm{~cm}$ and the ratio of long axis $>0.65$, The pathological complete response (pCR) was defined as no evident viable cancer cells [15]. Common Terminology Criteria for Adverse Events (CTCAE) Version 3.0 [16] was adopted to assess the adverse events of NAC.

\section{Follow-Up}

All patients were followed at outpatient clinics or contacted by a research nurse by phone. Over the first 3 years, the patients received clinical and radiological evaluation every 3 months. For the next 2 years, the patients were examined every 6 months. After 5 years, the patients were followed annually until death. The surveillance studies after surgery included chest CT scans, abdominal and cervical ultrasound. PET/CT, abdominal CT scan, endoscopy, brain MRI and ECT were performed depending on the patient's status.

\section{Statistical Analysis}

The SPSS 23.0 software for Windows (SPSS, Chicago, IL, USA) was used to perform the statistical analysis. The propensity score (PS) matched analysis was used to reduce the bias. PS was calculated to fit with variables, including age, sex, length of tumor, location of tumor, clinical T stage, clinical $\mathrm{N}$ stage and clinical TNM stage. The characteristics and pre-therapy data between the groups were compared using Student's t-test for all quantitative data and both a chi-square test and a Mann-Whitney U test for qualitative data. Categorical variables were compared by Fisher's exact test. The Kaplan-Meier method was adopted to analyze overall survival (OS) with a log-rank test. We reported survival rates and Hazard ratios with $95 \%$ confidence intervals (CIs). The OS was defined as the time from the operation in the surgery group and the first date of NAC to the death or most recent follow-up. A two-sided P value of 0.05 was defined as statistically significant.

\section{Results}

\section{Patient Characteristics}

Between 06/2011 and 12/2014, there were 279 consecutive patients who meet the criterion; 83 received NAC and 196 primary surgery. After PS matched analysis, there were 76 matched primary surgery patients (Figure 3). The characteristics of the 159 patients after PS matching were summarized in Table 1 . The primary data was comparable. The NAC group had much earlier pathological stage $(\mathrm{P}<0.001)$.

Table 1. Characteristics and pre-therapy data of patients with ESCC after and before the propensity score matching

\begin{tabular}{|c|c|c|c|c|c|}
\hline \multirow[t]{2}{*}{ Characteristics } & \multirow{2}{*}{$\begin{array}{l}\text { Number } \\
(\%)\end{array}$} & \multicolumn{4}{|c|}{ Different strategy } \\
\hline & & $S(n=76)$ & $\begin{array}{l}\text { NAC+S } \\
(n=83)\end{array}$ & $x^{2 / F}$ & $\begin{array}{l}p \\
\text { Value }\end{array}$ \\
\hline \multicolumn{6}{|c|}{$\begin{array}{l}\text { Characteristics and pre-therapy data of patients with ESCC after the propensity } \\
\text { score matching in } 159 \text { patients }\end{array}$} \\
\hline Age $Y($ Mean $\pm S D)$ & & $60.47 \pm 0.973$ & $60.6 \pm 0.824$ & 2.719 & 0.101 \\
\hline Range & & $40-74$ & $42-81$ & & \\
\hline Gender N (\%) & & & & 0.003 & 0.955 \\
\hline Male & $130(81.8)$ & $62(81.6)$ & $68(81.9)$ & & \\
\hline Female & $29(18.2)$ & $14(18.4)$ & $15(18.1)$ & & \\
\hline Location N (\%) & & & & 2.476 & 0.290 \\
\hline Upper thoracic & $35(22.0)$ & 15(19.7) & $20(24.1)$ & & \\
\hline Middle thoracic & $71(44.7)$ & $31(40.8)$ & $40(48.2)$ & & \\
\hline Lower thoracic & $53(33.3)$ & $30(39.5)$ & $23(27.7)$ & & \\
\hline $\begin{array}{l}\text { Clinical TNM staging } 7^{\text {th }} \\
\text { ed N }(\%)\end{array}$ & & & & 0.297 & 0.586 \\
\hline cStage II & $78(49.1)$ & $39(51.3)$ & $39(47.0)$ & & \\
\hline cStageIII & $81(50.9)$ & $37(48.7)$ & $44(53.0)$ & & \\
\hline Adjuvant therapy & & & & $<0.001$ & 0.982 \\
\hline Yes & $94(59.1)$ & $45(59.2)$ & $49(59.0)$ & & \\
\hline No & $65(40.9)$ & $31(40.8)$ & $34(41.0)$ & & \\
\hline Adjuvant radiation & & & & 0.202 & 0.653 \\
\hline Yes & 19(11.9) & $10(13.2)$ & $9(10.8)$ & & \\
\hline No & $140(88.1)$ & $66(86.8)$ & $74(89.2)$ & & \\
\hline $\begin{array}{l}\text { Pathological TNM } \\
\text { staging } 7^{\text {th }} \text { ed } N(\%)\end{array}$ & & & & & $<0.001^{a}$ \\
\hline $\mathrm{pCR}$ & 17(10.7) & NA & $17(20.5)$ & & \\
\hline I & $14(8.8)$ & $6(7.9)$ & $8(9.6)$ & & \\
\hline II & $74(46.5)$ & $42(55.3)$ & $32(38.6)$ & & \\
\hline III & $53(33.3)$ & $27(35.5)$ & $26(31.3)$ & & \\
\hline IV & $1(0.6)$ & $1(1.3)$ & 0 & & \\
\hline
\end{tabular}

\section{Clinical Effect of Neoadjuvant Chemotherapy}

CR to NAC were observed in 16 (19.3\%) patients, partial responses (PR) in $48(57.8 \%)$ patients, SD in 17 $(20.5 \%)$, and progressive disease (PD) in $2(2.4 \%)$. Therefore, the overall clinical response rate was $77.1 \%$. During the same period, there were 6 excluded NAC patients. One patient after NAC (clinical effect, stable disease, $\mathrm{SD}$ ) underwent $\mathrm{R} 1$ resection because of the invasion of aorta. Five patients didn't received surgery after NAC; three got progressive disease (PD) clinically and underwent definitive chemoradiotherapy. Two got clinical complete response (CR) after $\mathrm{NAC}$, refused to do surgery and accepted definitive chemoradiotherapy. 


\section{Toxicity of Neoadjuvant Chemotherapy}

Most of the patients received 2 cycles of NAC $(86.7 \%)$. Paclitaxel + NDP were the most adopted chemotherapy regimen $(55.4 \%)$. Table 2 showed the toxicity of NAC. One $(1.2 \%)$ patient had grade 4 leukopenia, $8(9.6 \%)$ had grade 3 leukopenia, and 33 $(39.8 \%)$ had grade 1 leukopenia. None of the patients had grade 4; hepatorenal and gastrointestinal toxicity. Only 3 patients adjusted the dose because of side effects.

Table 2. Side effects of NAC

\begin{tabular}{llllll}
\hline Toxicity & $\begin{array}{l}\text { Grade 0 } \\
\text { N (\%) }\end{array}$ & $\begin{array}{l}\text { Grade 1 } \\
\text { N (\%) }\end{array}$ & $\begin{array}{l}\text { Grade 2 } \\
\text { N (\%) }\end{array}$ & $\begin{array}{l}\text { Grade 3 } \\
\text { N (\%) }\end{array}$ & $\begin{array}{l}\text { Grade 4 } \\
\text { N (\%) }\end{array}$ \\
\hline $\begin{array}{l}\text { Leukopenia } \\
\begin{array}{l}\text { Gastrointestinal } \\
\text { symptoms }\end{array}\end{array}$ & $39(47)$ & $33(39.8)$ & $8(9.6)$ & $2(2.4)$ & $1(1.2)$ \\
$\begin{array}{l}\text { Hepatorenal } \\
\text { disorder }\end{array}$ & $43(51.8)$ & $31(37.3)$ & $11(13.3)$ & $4(4.8)$ & 0 \\
\hline a, Common Terminology Criteria for Adverse Events Version 3.0; N, number.
\end{tabular}

Table 3. Operation data for both groups

\begin{tabular}{llll}
\hline Variable & $\begin{array}{l}\text { NAC+S } \\
(\mathbf{n}=83)\end{array}$ & $\mathbf{S ~ ( n = 7 6 )}$ & $p$ Value \\
\hline Operation time(minutes), Mean \pm SD & $227.94 \pm 39.57$ & $239.74 \pm 47.293$ & 0.089 \\
EBL (ml), median (range) & $100(50-400)$ & $70(30-400)$ & 0.093 \\
Total nodes collected, median (range) & $16(15-69)$ & $16(15-42)$ & 0.608 \\
$\begin{array}{l}\text { Pathologically positive lymph nodes, } \\
\text { median (range) }\end{array}$ & $0(0-28)$ & $0(0-9)$ & 0.582 \\
Postoperative days, median (range) & $9(5-30)$ & $8(5-32)$ & 0.052 \\
Hospital mortality, (\%) & 0 & 0 & $\mathrm{NA}$ \\
Complication, N (\%) & $20(24.1)$ & $19(25.0)$ & 0.895 \\
Pneumonia & $11(13.3)$ & $9(11.8)$ & 0.789 \\
Arrhythmia & $7(8.4)$ & $6(7.9)$ & 0.901 \\
Anastomotic leakage & $2(2.4)$ & $1(1.3)$ & $>0.99$ \\
Incision infection & $4(4.8)$ & $2(2.6)$ & 0.759 \\
Recurrent laryngeal nerve injury & $16(19.3)$ & $13(17.1)$ & 0.723 \\
Chylothorax & 0 & $1(1.3)$ & 0.478 \\
\hline
\end{tabular}

NAC, neoadjuvant chemotherapy; S, surgery; EBL, estimated blood loss; $\mathrm{N}$, number; NA, not applicable; a Statistically significant $(p<0.05)$.

Table 4. Response evaluation of the patients with ESCC to NAC

\begin{tabular}{lll}
\hline Characteristics & Variable & Absolute value (\%) \\
\hline Radiologic response & CR & $16(19.3)$ \\
& PR & $48(57.8)$ \\
& SD & $17(20.5)$ \\
Pathologic response & PD & $2(2.4)$ \\
& CR & $17(20.5)$ \\
Down staging & other & $66(79.5)$ \\
& Yes & $33(39.8)$ \\
\hline
\end{tabular}

ESCC, esophagus squamous cell carcinoma; CR, complete response; PR, partial response; $\mathrm{SD}$, stable disease; $\mathrm{PD}$, progressive disease.

\section{Surgical Outcomes and Pathological Effects}

The surgical data were shown in Table 3. There was no significantly difference between the groups. Table 4 showed the clinical response to NAC. Based on RECIST 1.0 [14], 19.3\% of patients had CR, 57.8\% had PR, 20.5\% had SD and $2.4 \%$ had PD. The pathological response rate (pCR) was $20.5 \%$.

\section{Survival}

The median follow-up for all of the patients was 36 months (ranging from 6 to 68). Figure 2 described the OS of patients between the two groups with the Kaplan-Meier method, and a log-rank test was used. The five-year estimated OS rates for the NAC group and surgery group were $71.7 \%$ and $45.3 \%$, respectively. The mean survival time for the NAC group was 54.359 months (log-rank, 95\% CI, 50.417 to 58.301). The mean survival period was 49.353 months for the primary surgery group (log-rank, 95\% CI, 43.770 to 54.936 ). The estimate median survival time for surgery group was 51 months. However, the median survival time for the NAC group has not yet been reached. Seventeen pCR patients were all alive. Only one of them had recurrence in lung in 56 months after the first day of NAC.

\section{Discussion}

Our study demonstrated that NAC with TP followed by MIE with total two-field lymphadenectomy improved OS comparing with primary surgery. There are 2 possible reasons for the better NAC results. First, NAC-TP got a high clinical $(77.1 \%)$ and pathological $(20.5 \%)$ response rate. Second, the standarlized two-field lymphadenectomy MIE. In a study assessing the outcome of NACR followed by surgery, en bloc esophagectomy was compared with a transhiatal esophagectomy. The more radical surgery was an independently better survival factor, regardless of the response to NACR [17]. Another study on NACR for patients with ESCC showed that the degree of lymph node dissection significantly influenced the OS [18]. Compared with NACR, NAC didn't have the effect for local control. The quality of the operation was more important in NAC than NACR for EC [19]. In the 3 large multicenter clinical trials on NAC, too little attention was given to standardize the surgical procedure [19]. All the cases included in our study represent the results of a single surgeon's experience. That was easy to standardize surgical procedure.

The side effects toxicity of TP-NAC was tolerable. Hepatorenal disorder (grade 1) was the most common side effect (44.6\%) in the present study. Only $1(1.2 \%)$ patient had Grade 4 bone marrow depression. The vomiting and stomatitis were another common side effect, occurring in $4.8 \%$ of the patients (4 cases). The adverse effects of weekly paclitaxel and a 21-day cycle of cisplatin were mild. Due to the overall mild non-hematological and hematological toxicities, the weekly TP regimen was tolerable for many patients. 


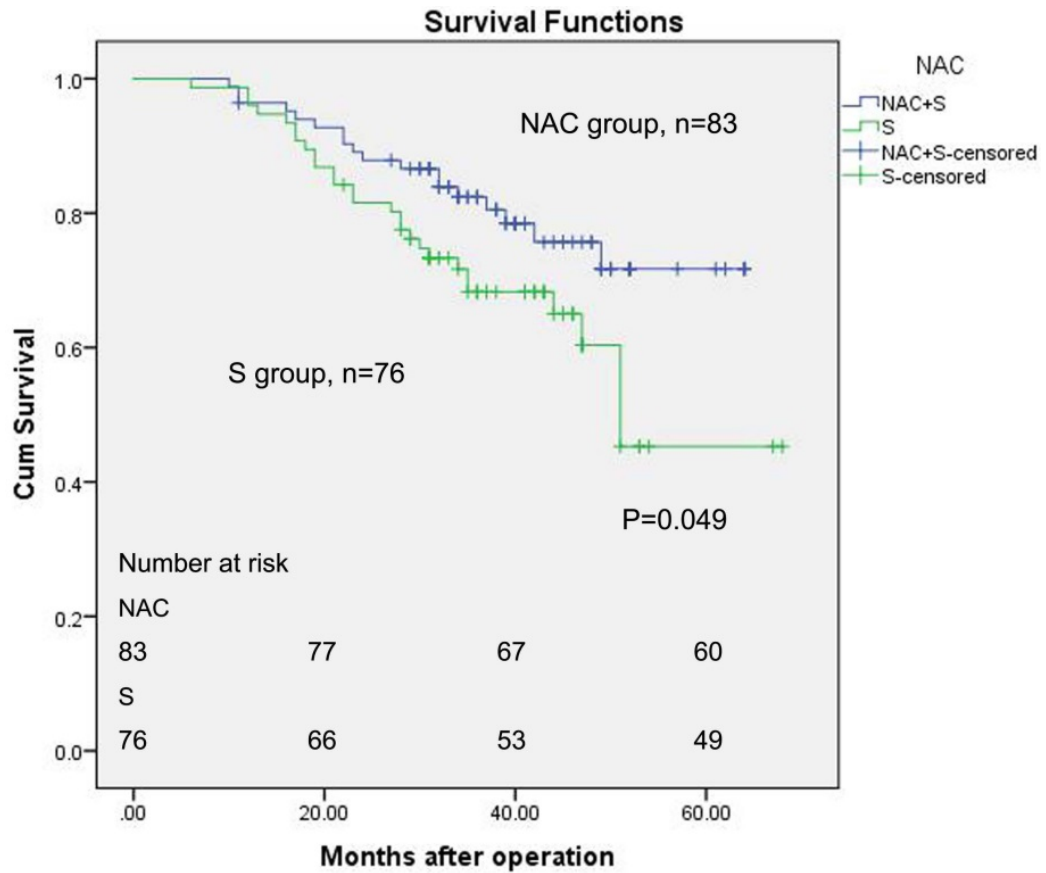

Figure 2. Kaplan-Meier survival analysis of ESCC patients $(n=159)$. The survival rate of patients in the NAC group was higher than patients in the surgery alone group (log-rank test, $P=0.049)$.

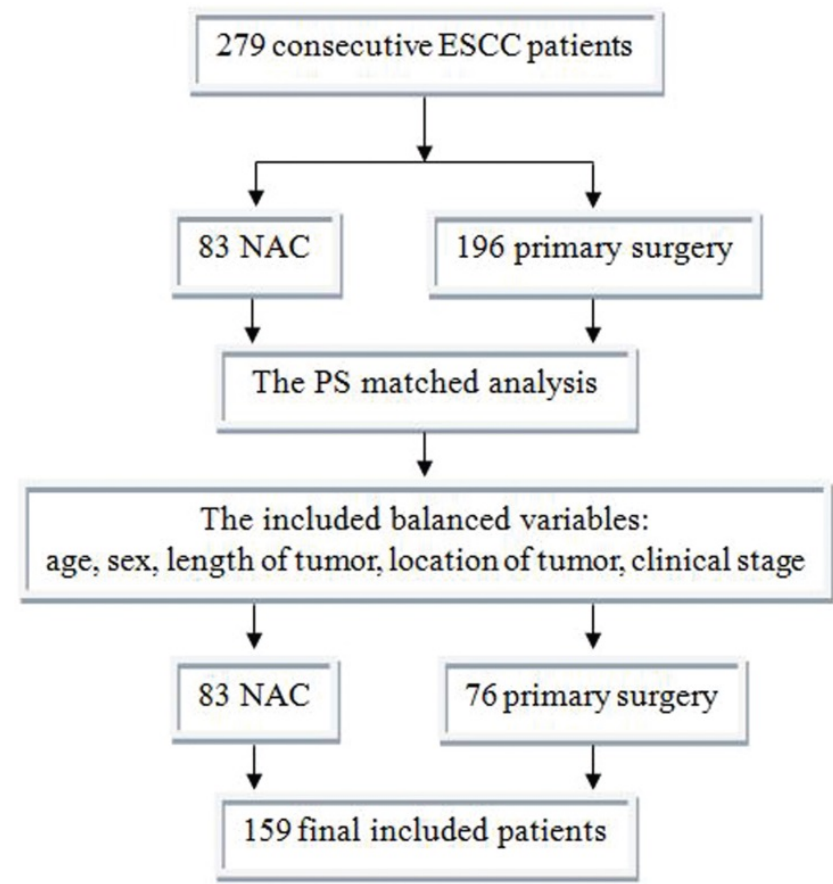

Figure 3. Between $06 / 2011$ and $12 / 2014$, there were 279 consecutive patients who meet the criterion; 83 received NAC and 196 primary surgery. After PS matched analysis, there were 76 matched primary surgery patients. Finally, there were 159 patients included in this study. ESCC, esophageal squamous cell carcinoma; NAC, neoadjuvant chemotherapy; PS, propensity score.

Esophageal surgery is usually associated with a high postoperative morbidity and mortality. However, our data shown the postoperative mortality was zero. After NAC, the incidence of postoperative morbidity and mortality has not increased among patients compared to patients treated by primary surgery. There was no significant difference in complication rate between two groups. MIE after NAC was safe.

The rates of $\mathrm{pCR}$ in patients treated with NAC ranged from $2 \%$ to $17 \%$ in previous reports of this combined strategy [11], whereas we achieved a pCR rate of $20.5 \%$ for primary tumors and the lymph nodes, which was relatively high. In previously reports, NACR could achieve a $20 \%-40 \%$ pCRT rate. The high $\mathrm{pCR}$ rate might be explained by the fact that the TP regime was adopted in this study, whereas in the past CF was mostly adopted. More and more studies have reported that paclitaxel cell toxicity is effective for treating squamous cell carcinoma (SCC) [20]. Based on the retrospective data and clinical experience, weekly paclitaxel was tolerable, might have been related to the high $\mathrm{pCR}$ rate. As a retrospective study, there must be some selection bias, which may also have contributed to the high $\mathrm{PCR}$ rate. That was also the reason why we wanted to start a RCT.

The ESCC mostly occurs in less-developed regions of the world. More than $50 \%$ of the ESCC cases were located in China (53\%, 210000 cases) [1]. Henan province carried the highest burden of ESCC in China [21]. Based on huge patient number, in light of past trials and the results of our studies, we designed the multicenter RCT in China [22]. Compared with past trials, we emphasized standard surgical procedures and a standard range of lymphadenectomy. The recruitment of 528 cases will be done within 2.5 years ClinicalTrials.gov Identifier: 
NCT02395705. Hopefully, it will provide a conclusion for the using of NAC with total two-field lymphadenectomy for ESCC.

\section{Conclusions}

NAC with TP followed by MIE with total two-field lymphadenectomy is better than primary surgery in terms of OS without additional serious adverse events. This combined therapy might be a highly recommended treatment modality for patients with stage II/III ESCC.

\section{Acknowledgements}

This project was supported by the Henan provincial public health authority (Grant Number. 201501003), the Henan province ministry of education (Grant Number. 17A320048) and the Wu Jieping fund (Grant Number. 320.6799.15062 and 320.2730.1892).

\section{Ethics approval}

The study was approved by the Henan Cancer Hospital Ethics Committee, approval number: 2016ct081

\section{Authors' contributions}

Y. Z., and Y. L. had full access to all of the data in the study and takes responsibility for the integrity of the data and the accuracy of the data analysis. Y.Z., X. L., R. Z., Z. W., H. S., and S. L. contributed substantially to the study design, data analysis and interpretation, and the writing of the manuscript. Y. Z., Y. L., Z. W., H. S., and S. L.

\section{Competing Interests}

The authors have declared that no competing interest exists.

\section{References}

1. Siegel R, Desantis C, Jemal A: Colorectal cancer statistics, 2014. CA: a cancer journal for clinicians 2014, 64(2):104-117.

2. Kelsen DP, Ginsberg R, Pajak TF, Sheahan DG, Gunderson L, Mortimer J, Estes N, Haller DG, Ajani J, Kocha W et al: Chemotherapy followed by surgery compared with surgery alone for localized esophageal cancer. The New England journal of medicine 1998, 339(27):1979-1984.

3. Kelsen DP, Winter KA, Gunderson LL, Mortimer J, Estes NC, Haller DG, Ajani JA, Kocha W, Minsky BD, Roth JA et al: Long-term results of RTOG trial 8911 (USA Intergroup 113): a random assignment trial comparison of chemotherapy followed by surgery compared with surgery alone for esophageal cancer. Journal of clinical oncology : official journal of the American Society of Clinical Oncology 2007, 25(24):3719-3725.

4. Allum WH, Stenning SP, Bancewicz J, Clark PI, Langley RE: Long-term results of a randomized trial of surgery with or without preoperative chemotherapy in esophageal cancer. Journal of clinical oncology : official journal of the American Society of Clinical Oncology 2009, 27(30):5062-5067.

5. Medical Research Council Oesophageal Cancer Working G: Surgical resection with or without preoperative chemotherapy in oesophageal cancer: a randomised controlled trial. Lancet 2002, 359(9319):1727-1733.

6. van Hagen $\mathrm{P}$, Hulshof $\mathrm{MC}$, van Lanschot JJ, Steyerberg EW, van Berge Henegouwen MI, Wijnhoven BP, Richel DJ, Nieuwenhuijzen GA, Hospers GA, Bonenkamp JJ et al: Preoperative chemoradiotherapy for esophageal or junctional cancer. The New England journal of medicine 2012, 366(22):2074-2084.

7. Ando N, Iizuka T, Ide H, Ishida K, Shinoda M, Nishimaki T, Takiyama W, Watanabe $\mathrm{H}$, Isono $\mathrm{K}$, Aoyama $\mathrm{N}$ et al: Surgery plus chemotherapy compared with surgery alone for localized squamous cell carcinoma of the thoracic esophagus: a Japan Clinical Oncology Group Study--JCOG9204. Journal of clinical oncology : official journal of the American Society of Clinical Oncology 2003, 21(24):4592-4596.

8. Ando N, Kato H, Igaki H, Shinoda M, Ozawa S, Shimizu H, Nakamura T, Yabusaki H, Aoyama N, Kurita A et al: A randomized trial comparing postoperative adjuvant chemotherapy with cisplatin and 5-fluorouracil versus preoperative chemotherapy for localized advanced squamous cell carcinoma of the thoracic esophagus (JCOG9907). Annals of surgical oncology 2012, 19(1):68-74.

9. Gebski V, Burmeister B, Smithers BM, Foo K, Zalcberg J, Simes J: Survival benefits from neoadjuvant chemoradiotherapy or chemotherapy in oesophageal carcinoma: a meta-analysis. The Lancet Oncology 2007, 8(3):226-234

10. Kumagai K, Rouvelas I, Tsai JA, Mariosa D, Klevebro F, Lindblad M, Ye W, Lundell L, Nilsson M: Meta-analysis of postoperative morbidity and perioperative mortality in patients receiving neoadjuvant chemotherapy or chemoradiotherapy for resectable oesophageal and gastro-oesophageal junctional cancers. The British journal of surgery 2014, 101(4):321-338.

11. Zheng Y, Li Y, Liu X, Sun H, Wang Z, Zhang R: Reevaluation of Neoadjuvant Chemotherapy for Esophageal Squamous Cell Carcinoma: A Meta-Analysis of Randomized Controlled Trials Over the Past 20 Years. Medicine 2015, 94(27):e1102.

12. Rice TW, Blackstone EH, Rusch VW: 7th edition of the AJCC Cancer Staging Manual: esophagus and esophagogastric junction. Annals of surgical oncology 2010, 17(7):1721-1724.

13. Zheng $Y$, Li $Y$, Wang $Z$, Sun $H$, Zhang R: A video demonstration of the Li's anastomosis-the key part of the "non-tube no fasting" fast track program for resectable esophageal carcinoma. Journal of thoracic disease 2015, 7(7):1264-1268

14. Eisenhauer EA, Therasse P, Bogaerts I, Schwartz LH, Sargent D, Ford R, Dancey J, Arbuck S, Gwyther S, Mooney M et al: New response evaluation criteria in solid tumours: revised RECIST guideline (version 1.1). European journal of cancer (Oxford, England: 1990) 2009, 45(2):228-247.

15. Davies AR, Gossage JA, Zylstra J, Mattsson F, Lagergren J, Maisey N, Smyth EC, Cunningham D, Allum WH, Mason RC: Tumor stage after neoadjuvant chemotherapy determines survival after surgery for adenocarcinoma of the esophagus and esophagogastric junction. Journal of clinical oncology : official journal of the American Society of Clinical Oncology 2014, 32(27):2983-2990.

16. Kaplan DK, Whyte RI, Donnelly RJ: Oesophagogastrectomy using stapling instruments. European journal of cardio-thoracic surgery : official journal of the European Association for Cardio-thoracic Surgery 1988, 2(2):95-99.

17. Rizzetto C, DeMeester SR, Hagen JA, Peyre CG, Lipham JC, DeMeester TR: En bloc esophagectomy reduces local recurrence and improves survival compared with transhiatal resection after neoadjuvant therapy for esophageal adenocarcinoma. The Journal of thoracic and cardiovascular surgery 2008, 135(6):1228-1236.

18. Chao YK, Liu HP, Hsieh MJ, Wu YC, Liu YH, Yeh CH, Chang HK, Tseng CK: Lymph node dissection after chemoradiation in esophageal cancer: a subgroup analysis of patients with and without pathological response. Annals of surgical oncology 2012, 19(11):3500-3505.

19. Markar SR, Wiggins T, Ni M, Steyerberg EW, Van Lanschot JJ, Sasako M, Hanna GB: Assessment of the quality of surgery within randomised controlled trials for the treatment of gastro-oesophageal cancer: a systematic review. The Lancet Oncology 2015, 16(1):e23-31.

20. Hashiguchi T, Nasu M, Hashimoto T, Kuniyasu T, Inoue H, Sakai N, Ouchi K, Amano T, Isayama F, Tomita N et al: Docetaxel, cisplatin and 5-fluorouracil adjuvant chemotherapy following three-field lymph node dissection for stage II/III N1, 2 esophageal cancer. Molecular and clinical oncology 2014, 2(5):719-724

21. Chen W, Zheng R, Baade PD, Zhang S, Zeng H, Bray F, Jemal A, Yu XQ, He J: Cancer statistics in China, 2015. CA: a cancer journal for clinicians 2016, 66(2):115-132.

22. Zheng Y, Li Y, Liu X, Zhang R, Wang Z, Sun H, Liu S: A phase III, multicenter randomized controlled trial of neo-adjuvant chemotherapy paclitaxel plus cisplatin versus surgery alone for stage IIA-IIIB esophageal squamous cell carcinoma. Journal of thoracic disease 2017, 9(1):200-204. 